\title{
Embarazo repetido en la adolescencia: la realidad chilena
}

\author{
Electra González A. ${ }^{1 a}$ \\ ${ }^{1}$ Centro de Medicina Reproductiva y Desarrollo Integral del Adolescente (CEMERA), Facultad de Medicina, Universidad \\ de Chile.
}

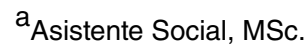

\section{RESUMEN}

Antecedentes: El embarazo repetido durante la adolescencia aumenta el riesgo para las madres de no completar sus estudios, tener más baja tasa de participación laboral y menores ingresos. Objetivo: Describir la magnitud del embarazo repetido en adolescentes y analizar los principales factores que se asocian a la reincidencia del embarazo adolescente en la Región Metropolitana. Método: Estudio descriptivo, analítico y transversal. La población del estudio fueron adolescentes embarazadas que se controlaron en el período Enero a Diciembre de 2007 en 8 consultorios de la Región Metropolitana. Los datos obtenidos desde las fichas clínicas. Variables estudiadas: edad, nivel educacional, relación de pareja, período intergenésico (PIG), paridad, edad gestacional al ingreso a control, uso de método anticonceptivo (MAC) y número de embarazos previos. Resultados: Del total de embarazadas del período en estudio, 852 (30\%) eran embarazadas adolescentes. De éstas el 15,6\% (133) correspondió a adolescentes reincidentes. El $5,2 \%$ de las reincidentes tenían 15 años y menos. El 67,7\% sólo cursó escolaridad básica. El 64,7\% estaban casadas o convivían. El 36,6\% presentó PIG de 11 meses y menos. El 6,8\% había tenido 2 o más gestaciones previas. El 59,4\% inició control prenatal después de las 12 semanas de gestación. El 73,5\% no usó MAC. Al relacionar PIG con escolaridad se observó que el 59,4\% de las adolescentes que tenían PIG de 11 meses y menos se concentraron en el nivel de menor escolaridad. Conclusiones: Las adolescentes estudiadas mostraron factores de alto riesgo los cuales deberían haber sido detectados previamente para fortalecer una estrategia de apoyo.

\section{PALABRAS CLAVE: Adolescentes, embarazo recurrente, riesgos biopsicosociales}

\section{SUMMARY}

Background: Repeat pregnancy during adolescence increases the risk for mothers not to complete their studies, have lower labor participation rate and less income. Objective: To describe the magnitude of recurrent pregnancy among adolescent females and analyze the main factors associated with recidivism of adolescent pregnancy in the Metropolitan Region. Method: A descriptive, analytical, cross-sectional study. The study population consisted of pregnant adolescents who were attended during January to December 2007 in eight primary care clinics in the northern sector of the metropolitan area. Data were collected from the medical records. The following variables, age, schooling level, relationship, interpregnancy period (IPP), parity, gestational age at admission control, contraceptive use and number of previous pregnancies, were studied. Results: Of all pregnant women in the study period, 852 (30\%) were pregnant teenagers. Of these $15.6 \%$ (133) corresponded to repeat adolescents. $5.2 \%$ of these women have 15 years and under. $67.7 \%$ attended only primary school. $64.7 \%$ are married or live together. $36.6 \%$ had IPP interval of 11 months and less. $6.8 \%$ had 2 or more previous pregnancies. $59.4 \%$ began prenatal care after 12 weeks gestation. $73.5 \%$ of these teenagers did not use birth control though that all of them were cited for their indication. When the IPP was related with schooling was observed that $59.4 \%$ of adolescents with IPP 11 months were 
concentrated in the lower level of education. Conclusions: The adolescents studied showed high risk factors which should have been previously identified to strengthen a support strategy.

\section{KEY WORDS: Adolescents, recurrent pregnancy, biopsychosocial risks}

\section{INTRODUCCIÓN}

El embarazo adolescente se asocia a mayores riesgos de control prenatal tardío e inadecuado y a peores resultados en el parto y puerperio cuando se comparan con mujeres adultas (1). El control prenatal inadecuado o tardío en estas mujeres también contribuye a resultados adversos para el hijo como mayor riesgo de mortalidad infantil, sobre todo dentro del primer año de vida como a más problemas de salud a lo largo de la vida del hijo. Se describe que a menor edad, relación de pareja (estado marital) y consumo de tabaco durante el embarazo, puede afectar un adecuado control prenatal como también afectar los resultados del parto (2-4). Posponer un segundo embarazo y tener un período intergenésico adecuado son cruciales para estas madres adolescentes ya que un corto período intergenésico es un factor de alto riesgo para los resultados de un parto. Se describe que por lo menos un intervalo de 24 meses se considera vital para restaurar la salud nutricional de la madre como para aminorar el estrés postparto que este conlleva. Se estima que un 30 a $50 \%$ de madres adolescentes que tuvieron su primer parto antes de los 18 años tienen un segundo entre los 12 a 24 meses posteriores (2-5).

Las madres adolescentes que tienen nuevos embarazos antes de los dos años enfrentan mayor riesgo de presentar pobres resultados en el parto y postparto que cuando fueron madres la primera vez. Varios estudios reportan que estas madres tienen mayores probabilidades de tener más bajos niveles de escolaridad, de vivir en la pobreza o depender de programas de beneficencia que las madres que tienen un solo hijo durante la adolescencia (2-5). Tener un segundo hijo durante la adolescencia aumenta el riesgo para las madres de no completar sus estudios, tener más baja tasa de participación laboral, menos ingresos, menos recursos económicos tanto para las madres como para sus hijos que aquellas adolescentes que posponen un segundo nacimiento $(6,7)$.

Alrededor del 20 a $25 \%$ de todos los nacimientos de mujeres adolescentes en los Estados Unidos correspondían a un segundo o tercer nacimiento. A la luz de estos resultados es que en diversos países se han desarrollado programas especializados en la atención de embarazadas adolescentes con el objetivo de evitar segundos embarazos. Estos programas incluyen manejo de caso con un amplio rango de servicios incluyendo visitas domiciliarias, uso apropiado de los servicios de salud tanto para la adolescente como para el hijo/a, servicios anticonceptivos, etc. Sin embargo la evaluación de estos programas no parece demostrar que éstos tengan una importante influencia en las adolescentes para demorar un segundo embarazo, por lo menos no para un grupo de ellas, ya que adolescentes que participaron en estos programas reportaron un segundo embarazo a los 6 meses en un $9 \%$, a los 12 meses en un $20 \%$, a los 18 meses un $29 \%$ y a los 24 meses un 39\% (8-12).

En Chile, un estudio reporta la historia de fecundidad en 295 mujeres adolescentes solteras que iniciaron su maternidad en la adolescencia (la mayoría había tenido su primer hijo entre los 16 y 18 años), que controlaron su primer embarazo en un programa especial dirigido a revertir las consecuencias de la maternidad adolescente en el sector norte de la Región Metropolitana. En ese programa se abordaron tanto los aspectos biomédicos como los aspectos psicosociales durante el período del embarazo y posterior a éste, tanto en la adolescente, su pareja y su familia incluyendo la visita domiciliaria como apoyo fundamental y también se apoyó fuertemente la continuidad escolar de quienes estaban estudiando al momento del embarazo, mostró que 8 años después del nacimiento del primer hijo, 229 (77,6\%) de estas mujeres volvieron a embarazarse, de estas $95(41,4 \%)$ repitieron el embarazo en la adolescencia (13).

Los factores que incidirían en un embarazo repetido en una adolescente serían los siguientes: a) Características de la adolescente madre tales como edad, bienestar psicológico, las adolescentes que eran muy jóvenes cuando ellas tuvieron su primer hijo tienen mayor riesgo de tener un segundo embarazo que las adolescentes mayores ya que estarían más tiempo expuestas al riesgo de actividad sexual $(14,15)$. Adolescentes con baja autoestima, que presenten problemas conductuales tales como uso de drogas están mayor riesgo de un rápido segundo embarazo (16). b) Desempeño escolar y metas educacionales que tenía la adolescente, antes del primer embarazo, las adolescentes que no han tenido un buen desempeño académico pueden ver terminadas sus aspiraciones y asumir que una fase de sus vidas comienza a la llegada de su primer hijo. Al contrario, las niñas que tenían un buen desempeño pueden querer volver al colegio después del nacimiento del hijo y pueden percibir 
mejor los mayores costos asociados a un nuevo hijo. Las adolescentes que abandonaron antes del primer embarazo o después del primer nacimiento tienen más riesgo de volver a embarazarse que aquellas que permanecen en el colegio $(17,18)$. c) Situación de pareja, casarse o iniciar convivencia con el progenitor de su primer hijo aumenta la probabilidad de volver a embarazarse y en un corto intervalo de tiempo $(17,18)$. d) El apoyo que la adolescente recibe de su familia de origen, principalmente de su madre, el embarazo reincidente ha sido asociado a bajos niveles de apoyo familiar, la reacción y la actitud de la madre y familia frente al primer embarazo, que la abuela se haga cargo de la crianza del hijo, esto último puede ser debido a que la madre adolescente descansa de las responsabilidades y desafíos de la maternidad. Se postula que es posible que teniendo el rol activo del cuidado personal del hijo sea un incentivo para que la adolescente use anticoncepción o se abstenga de la actividad sexual (18-21). e) Características de la familia de origen de la adolescente, reincidencia del embarazo en el corto plazo ha sido asociado a bajo nivel de escolaridad de la madre, bajos niveles de apoyo familiar, como también a características familiares como tipo de familia, antecedente familiar de embarazo en la adolescencia y pobreza $(22,23)$. f) Antecedente de abuso sexual, algunos estudios han reportado que un porcentaje importante de adolescentes que quedaron embarazadas tenían antecedente de abuso sexual y que es posible que este antecedente acreciente el riesgo de embarazo reincidente en el corto plazo (24-27). g) Factores que están asociados al primer embarazo, como menor edad de inicio de pololeo, menor edad inicio de la actividad sexual, mayor número de parejas sexuales $(2,3,26)$. h) El no uso o uso inconsistente de métodos anticonceptivos en el postparto, el acceso a servicios de anticonceptivos, en sí mismo, no disminuye las tasas de nacimiento durante la adolescencia. Pero si puede reducir estas tasas cuando es combinado con claras metas educacionales y apoyo provisto por la escuela y programas especiales. El no uso o mal uso de anticonceptivos está fuertemente asociado a factores tales como, la forma en que se entrega la información y se entrega el servicio, la forma en que estos son percibidos y la utilización de éstos por parte de la adolescente. Además, es importante considerar la actitud de la madre y de la pareja de la adolescente hacia los anticonceptivos que a su vez va a influir en la actitud de la adolescente hacia el uso de métodos anticonceptivos (28-36). i) Antecedente de aborto, se describe que la historia de abortos está asociada a los embarazos reincidentes en los dos primeros años (36).

El objetivo del estudio fue describir la magnitud del embarazo reincidente en adolescentes y analizar los principales factores que se asocian a la reincidencia del embarazo, en adolescentes consultantes en la atención primaria de la Región Metropolitana.

\section{PACIENTES Y MÉTODO}

Estudio descriptivo, analítico y transversal. La población del estudio estuvo constituida por adolescentes que controlaron su embarazo en el sistema de salud público del sector norte de la Región Metropolitana. Se seleccionaron 8 consultorios del área norte de la Región Metropolitana. Previo a la autorización de los respectivos directores, se realizó una revisión de todas las fichas clínicas correspondientes a todas las mujeres que controlaron embarazo en el período Enero a Diciembre de 2007. Luego se seleccionaron las fichas de las mujeres adolescentes. Finalmente se seleccionaron las fichas de aquellas adolescentes que cursaban un segundo o tercer embarazo. En función de la información disponible en las fichas se estudiaron las siguientes variables: edad, escolaridad, relación de pareja, periodo intergenésico (PIG), paridad, edad gestacional al ingresar al control prenatal, uso de método anticonceptivo post primer parto, embarazo reincidente. Los datos así obtenidos fueron ingresados a una base de datos para su análisis. Para los análisis uni y bivariado se utilizó el software STATA 10.0. Se consideró significativo un valor $p<0,05$.

\section{RESULTADOS}

El total de embarazadas del período en estudio fue de 2.796. De este total, 852 (30\%) correspondió a embarazadas adolescentes. De éstas 852 embarazadas adolescentes, el 15,6\% (n: 133) eran embarazos repetidos. El análisis descriptivo de los datos muestra que el 5,2\% tiene 15 años o menos al ingreso a control prenatal, un $30 \%$ entre 16 y 17 años y el 64,7\% entre 18 y 19 años. El 24\% de las adolescentes estaban casadas, un $41,4 \%$ convivía con su pareja, un 33,8\% permanecían solteras. En cuanto a la escolaridad el $67,7 \%$ solo llegó hasta enseñanza básica, un 32,3\% alcanzó algún curso de enseñanza media. El 35\% reportó consumo de tabaco (Tabla I). El 93,2\% había presentado una gestación previa mientras que un $6,8 \%$ reportó 2 o más embarazos previos. El 18,2 $\%$ de las gestaciones previas no terminó en parto. El $22 \%$ declara uno o más abortos previos. El $40,6 \%$ inició este control prenatal antes de las 12 semanas de gestación, 35,3\% lo hizo entre 12 y 18 semanas y un $24,1 \%$ lo hizo a las 19 semanas o más. El 36,6\% presentó un PIG de 11 meses y menos, el 30,9\% entre 12 y 24 meses y el $32,5 \%$, más de 24 meses. El 73,5\% de estas adolescentes reincidentes no usaron método anticonceptivo (Tabla II). Al relacionarse el PIG con escolaridad se observó que el $59,4 \%$ de las adolescentes que 
Tabla I

\section{CARACTERISTICAS SOCIODEMOGRAFICAS DE LAS ADOLESCENTES DEL ESTUDIO (133 EMBARAZOS ADOLESCENTES REPETIDOS)}

\begin{tabular}{lccc}
\hline Variable & Categorías & $\mathrm{n}$ & $\%$ \\
\hline Edad 8 (años) & $\leq 15$ & 7 & 5,2 \\
& $16-17$ & 40 & 30,0 \\
& $18-19$ & 86 & 64,8 \\
\hline Escolaridad & $1^{\circ}$ a $4^{\circ}$ Básico & 7 & 5,3 \\
& $5^{\circ}$ a $8^{\circ}$ Básico & 83 & 62,4 \\
& $1^{\circ}$ a $2^{\circ}$ Medio & 19 & 14,3 \\
& $3^{\circ}$ a $4^{\circ}$ Medio & 24 & 18,0 \\
\hline Estado Civil & Soltera & 45 & 33,8 \\
& Casada & 32 & 24,0 \\
& Convive & 55 & 41,4 \\
\hline Fuma & $\mathrm{Si}$ & 33 & 35,0 \\
& No & 100 & 75,0 \\
\hline
\end{tabular}

\section{Tabla II \\ CARACTERISTICAS OBSTETRICAS EN 133 EMBARAZOS ADOLESCENTES REPETIDOS}

\begin{tabular}{|c|c|c|c|}
\hline Variables & Categorías & $\mathrm{n}$ & $\%$ \\
\hline $\begin{array}{l}\text { Número de gesta- } \\
\text { ciones previas }\end{array}$ & $\begin{array}{c}1 \\
\geq 2\end{array}$ & $\begin{array}{c}124 \\
9\end{array}$ & $\begin{array}{c}93,2 \\
6,8\end{array}$ \\
\hline Partos previos & $\begin{array}{l}0 \\
1 \\
2\end{array}$ & $\begin{array}{c}24 \\
105 \\
3\end{array}$ & $\begin{array}{c}18,2 \\
79,5 \\
2,3\end{array}$ \\
\hline Abortos previos & $\begin{array}{l}0 \\
1 \\
2 \\
6\end{array}$ & $\begin{array}{c}103 \\
26 \\
2 \\
1\end{array}$ & $\begin{array}{r}78,0 \\
19,7 \\
1,5 \\
0,8\end{array}$ \\
\hline $\begin{array}{l}\text { Semanas de em- } \\
\text { barazo al ingreso a } \\
\text { control prenatal }\end{array}$ & $\begin{array}{c}<12 \\
12-18 \\
\geq 19\end{array}$ & $\begin{array}{l}54 \\
47 \\
32\end{array}$ & $\begin{array}{l}40,6 \\
35,3 \\
24,1\end{array}$ \\
\hline $\begin{array}{l}\text { Periodo intergené- } \\
\text { sico (meses) }\end{array}$ & $\begin{array}{c}<6 \\
6-11 \\
12-24 \\
>24\end{array}$ & $\begin{array}{l}20 \\
28 \\
41 \\
44\end{array}$ & $\begin{array}{l}15,1 \\
21,5 \\
30,9 \\
32,5\end{array}$ \\
\hline $\begin{array}{l}\text { Uso de anticoncep- } \\
\text { tivo post embarazo } \\
\text { anterior }\end{array}$ & $\begin{array}{l}\text { No uso } \\
\text { Con uso } \\
\text { No consignado }\end{array}$ & $\begin{array}{l}74 \\
27 \\
32\end{array}$ & $\begin{array}{l}55,6 \\
20,3 \\
24,1\end{array}$ \\
\hline $\begin{array}{l}\text { Tipo de anticon- } \\
\text { ceptivo usado }\end{array}$ & $\begin{array}{l}\text { Oral } \\
\text { Diu } \\
\text { Preservativo }\end{array}$ & $\begin{array}{c}9 \\
16 \\
2\end{array}$ & $\begin{array}{c}33,3 \\
59,3 \\
7,4\end{array}$ \\
\hline
\end{tabular}

tenían PIG de 11 meses y menos se concentran en el nivel de escolaridad de enseñanza básica $(p=0,00)$ (no mostrado en la Tabla). En cuanto a las características de las parejas se observó que el $19,6 \%$ de las parejas tenían 19 años y menos, $54,1 \%$ solo alcanzó escolaridad básica, el $91,2 \%$ tenía algún trabajo no calificado, el $55,4 \%$ con consumo de tabaco, un $42,9 \%$ con consumo de alcohol y $21 \%$ con consumo de drogas (Tabla III).

Tabla III

CARACTERISTICAS DE LAS PAREJAS DE LAS ADOLESCENTES

\begin{tabular}{llcc}
\hline Variables & Categorías & $\mathrm{n}$ & $\%$ \\
\hline Edad (años) & $\leq 19$ & 19 & 19,6 \\
& $20-24$ & 49 & 50,5 \\
& $\geq 25$ & 29 & 29,9 \\
\hline Estudios & Educación & 20 & 54,1 \\
& básica & & \\
& Educación & 17 & 45,9 \\
& media & & \\
\hline Actividad & Estudia & 8 & 8,8 \\
& Trabajo no & 83 & 91,2 \\
& calificado & & \\
\hline Consumo cigarrillos & Si & 36 & 55,4 \\
& No & 29 & 44,6 \\
\hline Consumo alcohol & Si & 27 & 42,9 \\
& No & 36 & 57,1 \\
\hline Consumo drogas & Si & 13 & 21,0 \\
& No & 49 & 79,0 \\
\hline
\end{tabular}

\section{DISCUSIÓN}

Los resultados del presente estudio muestran que el $15,6 \%$ del total de las adolescentes embarazadas del estudio repitieron el embarazo y un $5,2 \%$ tenía 15 años y menos, lo que es bastante superior a las cifras nacionales $(5,8 \%$ y $2,5 \%$, respectivamente) (37). Un 65,4\% estaba casada o convivía con el progenitor lo que reafirma lo encontrado en la literatura que muestra que la situación de pareja, casarse o convivir con el progenitor del primer hijo aumenta el riesgo de repetir embarazo en la adolescencia (17-19). Dos tercios de ellas solo cursó hasta enseñanza básica, otro factor de riesgo que la literatura muestra asociado a la reincidencia del embarazo adolescente (17-19). 
En relación a los antecedentes obstétricos se puede observar que el $22,2 \%$ de las gestaciones anteriores terminaron en aborto, estudios muestran que una historia de aborto está asociada a los embarazos reincidentes en los dos primeros años (31-35).

Llama la atención el $73,5 \%$ de no uso de métodos anticonceptivos post parto, descrito en la literatura como un importante factor asociado a la repetición del embarazo (30-31), en circunstancias que en los registros de las historias clínicas aparecían citadas a programas de planificación familiar. De las pocas que usaron anticonceptivos orales es probable que su uso fuera inconsistente. Las adolescentes suelen calificarse como "olvidadizas" para un uso consistente de los ACOs. Cabe preguntarse también cómo se entregó la información, y o la entrega de anticonceptivos y como lo percibió la adolescente. Es preocupante también que 16 de ellas se embarazaron con uso de DIU.

Los PIG que presentaron estas adolescentes son alarmantes ya que sólo un tercio de ellas tuvo un PIG recomendado como adecuado, es decir, después de 24 meses. La mayoría presentó PIG muy cortos lo que se asocia fuertemente a consecuencias adversas en la salud tanto de la madre como del recién nacido.

Lo que es preocupante en este estudio es que adolescentes que ya habían estado en el sistema de salud, han tenido oportunidad de controlarse, han tenido acceso a consejería y a la provisión de métodos anticonceptivos en el sistema de salud primaria, sin embargo no lograron usarlos.

Las estadísticas nacionales en relación a segundo embarazo adolescente, objetivado como segundo hijo, muestran que en el 2009, 62 adolescentes menores de 15 años tuvieron un segundo hijo (5,8\%) cifra que disminuyó a 24 (2,5\%) el 2010 (37). En el grupo de adolescentes de 15 a 19 años los porcentajes de segundo hijo oscilan entre 10 a 14\% entre el 2007 y 2012 (37). Esta disminución del embarazo repetido en adolescentes puede ser atribuido a que la red pública de salud ha mejorado notoriamente sus servicios a través del programa de salud integral adolescente, a que en varios hospitales se ha implementado un mejor acceso al uso de métodos anticonceptivos de larga duración (implante) en el postparto inmediato.

$\mathrm{Si}$ bien las autoridades han mejorado las estrategias para alcanzar a estas adolescentes en el postparto inmediato, desarrollando nuevas estrategias dirigidas a la prevención del segundo embarazo priorizando un mayor y mejor acceso a métodos anticonceptivos de larga duración durante el postparto inmediato, se hace indispensable realizar estudios cualitativos para investigar en mayor profundidad en los otros factores que inciden en el embarazo repetido en adolescentes.
Limitaciones del estudio: Debido a que los datos fueron obtenidos a través de una fuente de información secundaria como fueron las historias clínicas, no permitió la exploración de otras variables que aparecen asociadas a la reincidencia del embarazo en adolescentes como características de las familias de origen, edad de inicio de la actividad sexual, historia de abuso sexual, etc. Este estudio fue realizado casi 10 años atrás, sin embargo, da cuenta que la mayoría de los factores que inciden en el embarazo repetido en adolescentes continúan estando presentes en la actualidad.

\section{CONCLUSIÓN}

El número de embarazos repetidos en la adolescencia ha disminuido pero es igualmente preocupante en salud pública dada la compleja problemática social que implica tanto para la madre adolescente como para sus hijos. Es importante continuar desarrollando nuevas estrategias priorizadas en la prevención del segundo embarazo que consideren todos los factores que se asocian a este evento.

\section{REFERENCIAS}

1. Donoso E, Becker J, Villarroel L. Birth rates and reproductive risk in adolescents in Chile, 1990-1999. Rev Panam Salud Publica/Pan Am J Public Health 2003;14(1):3-8.

2. Fraser C, Brockert J, Ward R. Association of young maternal age with adverse reproductive outcomes, $\mathrm{N}$ England J Med 1995;332:1113-8

3. Leland N, Petersen D, Braddock M, Alexander G. Variations in pregnancy outcomes by race among 10-14 year old mothers in the United States, Public Health Report 1995;110(1):53-8.

4. Wiemann C, Berenson A, Garcia del Pino L, McCombs S. Factors associated with adolescents risk for late entry into prenatal care. Fam Plann Perspec, 1997;29(6):273-6.

5. Langer A. El embarazo no deseado: Impacto sobre la salud y la sociedad en América Latina y el Caribe. Rev Panam Salud Pública 2003;11:192-204.

6. De Sousa-Mata A, Araujo-Lemos C, Lira-Ferreira C, Pereira-Braga L, Chaves-Maia E. Fatores de risco na repetição de gravidez na adolescência, Rev Colombiana de Psicología 18(2):167-75.

7. Acuña $\mathrm{H}$, Ochoa $\mathrm{Y}$. Complicaciones materno perinatales en adolescentes con reincidencia de embarazo, estudio caso control. Instituto Nacional Materno Perinatal 2006. Disponible en: http:// cybertesis.unmsm.edu.pe/bitstream/cybertesis/2753/1/ Acu\%C3\%B1a_uh.pdf.

8. Sims K, Luster T. Factors related to early subsequent pregnancies and second births among adolescent mothers in a family support program, J Family Issues 2002;23:1006-31.

9. Sangalang B, Barth R, Painter J. First-birth outcomes and timing of second births: A statewide case management program for adolescent mothers. Health of Social Work 2006;31(1):54-63. 
10. Klerman L, Baker B, Howard G, Second births among teenage mothers: program results and statistical methods. J Adolesc Health 2003;32:452-5.

11. Falk G, Ostlund L, Magnuson A, Schollin J, Nilsson $\mathrm{K}$, Teenage mothers- a high-risk group for new unintended pregnancies. Contraception 2006;74:4715.

12. Manlove J, Mariner C, Romano A. Subsequent fertility among teen mothers: Longitudinal analyses of recent national data. J Marriage Fam 2000;62:430-48.

13. González E, Molina T, Molina T, Historia de fecundidad en mujeres que iniciaron su maternidad en la adolescencia. Libro Resumen del IV Congreso Latinoamericano de Ginecología Infanto-Juvenil,1995, pp 96-97.

14. Persona L, Kaluda A, Tarallo M, Perfil de adolescentes com repetição da gravidez atendidas num ambulatorio de prenatal, Rev Latino Americana de Enfermagem 2004;12(5):745-50.

15. Kalmuss DS, Namerow PB, Subsequent childbearing among teenage mothers: the determinants of a closely spaced second birth, Fam Plan Perspect 1994;26(4):149-53.

16. Gillmore M, Lewis S, Lorh M, Spencer M, White R, Repeat pregnancies among adolescent mothers. J Marriage Fam 1997;59(3):536-50.

17. Pfitzner M, Hoff C, McElligott K. Predictors of repeat pregnancy in a program for pregnant teens. J Pediatr Adolesc Gynecol 2003;16:77-81.

18. Atkin L, Alatorre-Rico J. Pregnant again? Psychosocial predictors of short-interval repeat pregnancy among adolescent mothers in Mexico City. J Adol Health 1992;13(8):700-6.

19. Musick J. Young, poor, and pregnant: the psychology of teenage motherhood. N Eng J Med 1993;329 (21):1587-8.

20. Boardman L, Allsworth J, Phipps M, Lapane K. Risk factors for unintended versus intended rapid repeat pregnancies among adolescents, J Adolesc Health 2006;39:597-603.

21. Steven-Simon C, Kelly L, Singer D, Nelligan D. Reasons for first teen pregnancies predict the rate of subsequent teen conceptions. Pediatrics 1998;101(1):1-6.

22. Stevens-Simon C, Dolgan J, Kelly L, Singer D, The effect of monetary incentives and peer support groups on repeat adolescent pregnancies. A randomized trial of the Dollar- a-Day Program. JAMA 1997;26(12):97782.

23. Rowlands S. Social predictors of repeat adolescent pregnancy and focused strategies. Best Pract Res Clin Obstet Gynaecol 2010;24(5):605-16.

24. Oliveira V, Costa A, Lima F, Oliveira V, Brandao A, Marinho $L$, Mendes R. Via de parto em gestações sucessivas em adolescentes: Estudo de 714 casos. RBGO 2004, 26(9):703-7.

25. Ownbey M, Ownbey J, Cullen J. The effects of a healthy families home visitation program on rapid and teen repeat births. Child Adol Soc Work 2011;28:43958.

26. Boyer D, Fine D. Sexual abuse as a factor in adolescent pregnancy and child maltreatment. Fam Plan Perspect 1992;24(1):4-19.

27. Butler J, Burton L, Rethinking teenage childbearing: Is sexual abuse a missing link. Family Relations 1990;39(1):73-80.

28. Núñez- Urquiza R, Hernández-Prado B, GarciaBarrios C, González D, Walker D. Embarazo no deseado en adolescentes y utilización de métodos anticonceptivos postparto. Rev Salud Publica México 2003;45:92-102.

29. Han L, Teal BS, Sheeder J, Tocce K. Preventing repeat pregnancy in adolescents: is immediate postpartum insertion of the contraceptive implant cost effective? Am J Obstet Gynecol 2014;24:1-7.

30. González E, Caba F, Molina T, Sandoval J, Montero A, Oyarzún P, Molina R, Meneses R. Factores familiares asociados al uso de anticonceptivos en mujeres adolescentes sexualmente activas. Rev Sogia 2005;12(1):9-16.

31. Steven-Simon C, Kelly L, Singer D. Absence of negative attitudes toward childbearing among pregnant teenagers. A risk factor for a rapid repeat pregnancy? Arch Pediatr Adolesc Med 1996;150(10):1037-43.

32. Tocce K, Sheeder J, Teal S. Rapid repeat pregnancy in adolescents: do immediate postpartum contraceptive implants make a difference? Am J Obstet Gynecol 2012;481-8.

33. Usher-Seriki K, Smith B M, Callands T. Motherdaughter communication about sex and sexual intercourse among middle to upper class African American girls. J Family Issues 2008;29(7):901-7.

34. Thompson L, Spanier G. Influence of parents, peers and partners on the contraceptive use of college men and women. J Marriage Family 1978;40(3):481-92.

35. González E, Encina C, Gómez M, Molina T. Factores psicosociales que se asocian a la reincidencia del embarazo en adolescentes chilenas. Contribución a Congreso Sogia. Rev Sogia 2003;10:71-2.

36. Paukku M, Quan J, Darney P, Raine T. Adolescents' contraceptive use and pregnancy history: Is there a pattern? Obstet Gynecol 2003;101(3):534-8.

37. Burdiles P, Santander S. Situación actual del embarazo adolescente en Chile. Documento Programa Salud integral de adolescentes y jóvenes, Depto. Ciclo vital. Minsal, Julio 2013, pp 1-53 